\title{
Providencia alcalifaciens patógeno emergente causante de diarreas
}

\author{
Jorge Mauricio Montero García \\ Licenciado en Microbiología y Química Clínica. Microbiólogo Químico Clínico. Hospital Los Chiles, Sección bacte- \\ riología, Caja Costarricense de Seguro Social; mont26@hotmail.com
}

Recibido: 02 febrero 2016

Aceptado: 26 abril 2016

\begin{abstract}
RESUMEN
Providencia alcalifacienses un microorganismo de la flora intestinal normal, perteneciente a la familia Enterobacteriacea. Estudios a lo largo del tiempo han relacionado a este microorganismo con cuadros de diarrea principalmente en niños. Investigaciones recientes han demostrado algunos mecanismos por los cuales esta bacteria puede ocasionar cuadros de gastroenteritis tales como: la capacidad invasora de esta bacteria, presencia de genes $c d t$ capaces de codificar para la toxina CDT y presencia de la Manganeso Súper Óxido Dismutasa, enzima que le permite sobrevivir intracelularmente.
\end{abstract}

Palabras clave: Diarrea, gastroenteritis, factores de virulencia.

\begin{abstract}
Providencia alcalifacienes is a microorganism of the normal intestinal flora, belonging to the family Enterobacteriaceae. Studies over the years have related to this organism mainly with cases of diarrhea in children. Recent research has shown some mechanisms by which these bacteria can cause gastroenteritis boxes such as invasiveness of the bacteria, the presence of $c d t$ genes capable of coding for the CDT toxin and the presence of manganese superoxide dismutase, an enzyme that allows you survive intracellularly.
\end{abstract}

Key words: Diarrhea, gastroenteritis, virulence factors.

\section{Introducción}

El género Providencia, pertenece a la familia Enterobacteriacea y consta de 8 especies, 1lamadas P. alcalifaciens, $P$. stuartii, $P$. rettgeri, $P$. rustugianii, $P$. beinbachae, $P$. vermicola, $P$. sneebia y P. Burhodogranariea (1). Los miembros de este género son anaerobios facultativos, móviles con flagelos peritricos (2). Este género es de flora intestinal normal (3) y se considera un comensal gastrointestinal (4), por lo que la mayoría de laboratorios clínicos no lo consideran como un agente potencial que causa diarreas (5) sin embargo, hay estudios que relacionan a Providencia alcalifaciens con casos de gastroenteritis en humanos ocasionando cuadros de diarrea, fiebre y vómito en países como Brasil, Japón, República Checa, Gran Bretaña y Kenia(4, 5, 7, 8, 9, 10). Uno de los medios de cultivo descrito capaz de diferenciar el crecimiento de Providencia alcalifaciens es el medio PAM (Providencia alcalifaciens medium) en el cual las colonias son rojas, mientras que las colonias de otras bacterias son blancas o amarillo limón debido a la fermentación de uno o varios de los azúcares presentes (xilosa, manitol y galactosa) (6).

\section{Patogenicidad}

Si bien es cierto, el mecanismo por el cual Providencia alcalifaciens provoca los cuadros de diarrea no está bien dilucidado, varios estudios han demostrado la capacidad de esta bacteria de invadir líneas celulares como HeLa, HEp-2 y Caco-2 atribuyendo esta capacidad como un factor de virulencia $(7,11,12)$. Mathan et al aportan más evidencia con algunos modelos de virulencia que demostraron dos mecanismos por los cuales la bacteria puede ingresar al interior de la célula. Uno de ellos es mediado por endocitosis directa asociada a polimerización de componentes del citoesqueleto y el otro mecanismo es por medio de la disrupción de las 
uniones estrechas, lo cual le permite a la bacteria proliferar en el espacio intercelular (2).

Murata et al, estudiaron la enteropatogenicidad de la cepa causante de un brote de diarrea en un jardín de niños en Japón en 1996, la cual inocularon a nivel intestinal en conejos de laboratorio. Cada aislamiento causó un aumento moderado en la acumulación de fluido a nivel intestinal, y cambios histopatológicos como inflamación de la mucosa, infiltración de neutrófilos y eosinófilos en la lámina propia, criptas y células endoteliales de los vasos sanguíneos, además se observó una distorsión de la arquitectura de las vellosidades característico de enteritis aguda (10). Otro factor de virulencia que se ha descrito, es la presencia de la Manganeso Súper Óxido Dismutasa (Mn-SOD). La Súper Óxido Dismutasa (SOD) convierte los aniones superóxido producidos por el estrés oxidativo a oxígeno molecular y peróxido de hidrógeno, los cuales posteriormente son metabolizados por catalazas y peroxidasas. Hay investigaciones que sugieren que las SOD juegan un rol importante en la virulencia de diferentes microorganismos durante el estallido respiratorio seguido de la fagocitosis. Este hallazgo en $P$. alcalifaciens sugiere que la Mn-SOD está involucrada en el proceso invasivo lo cual le permite sobrevivir intracelularmente en los macrófagos de manera similar a Salmonella o Yersinia (14).

Por otro lado, investigaciones recientes han revelado la presencia de genes $c d t$ los cuales codifican para una toxina de distendimiento endotelial (CDT por sus siglas en inglés), similar a la que produce Escherichia coli (15). Esta toxina provoca elongación celular, distensión de las células y un bloqueo irreversible en el ciclo de división celular en fase G2/M lo cual genera muerte celular $(15,13)$. Cepas de E. coli productoras de esta toxina se han aislado en pacientes que han sufrido cuadros de diarrea sanguinolenta(16). Se cree que el locus puede ser adquirido horizontalmente a través de fagos o por medio de transposones, curiosamente la CDT de Providencia alcalifaciens (PaCDT) muestra cierta homología con la CDT de Shigella boydii (16). La comprobación de algunos de los factores de virulencia de Providencia alcalifaciens, demuestra cómo esta bacteria es capaz de producir cuadros de diarrea y muestra la importancia que tiene como patógeno emergente.

\section{Conclusiones}

A pesar de la evidencia científica que apoya el hecho de que Providencia alcalifaciens es un patógeno emergente causante de diarreas en niños, en nuestro sistema de salud no se hace una búsqueda activa de este microorganismo lo que podría ocasionar que no se diagnostiquen cuadros de diarrea debido a que este microorganismo aún se considera flora normal. Por otro lado, sería interesante hacer una investigación de este microorganismo y secuenciar las cepas que circulan en nuestro país para determinar la presencia de los genes $c d t$ que codifican la toxina CDT capaz de provocar cuadros de diarreas y determinar si efectivamente se están sub diagnosticando cuadros de diarrea por no darle la importancia requerida a esta bacteria.

\section{Referencias}

ALBERT, M., ALAM, K., ANSARUZZAMAN, M., ISLAM, M., RAHMAN, A., HAIDER, K., BHUIYAN, N., NAHAR, S., RYANA, S., MONTANARO, J. \& MATHAN, M. (1992). Pathogenesis of Providencia alcalifaciens-Induced Diarrhea. Infection and Immunity, 60(12), 5017-5024.

CHEM, X., KODAMA, T., LIDA, T. \& HONDA, T. (2007). Demostration and characterization of Manganese Superoxide Dismutase of Providencia alcalifaciens. Microbiology immunology, 51(10), 951-961.

CHLIBEK, R., JIROUS, J. \& BERAN, J. (2002). Diarrhea outbreak among Czech Army Field Hospital personnel caused by Providencia alcalifaciens. Journal Travel Medicine. 9, 151-152.

GUTH, B. \& PERELLA, E. (1996). Prevalence of invasive ability and other virulence-associated characteristics in Providencia alcalifaciens strains isolated in Sao Pablo, Brazil. Journal of Medicine Microbiology. 45, 459-462.

JANDA, J., \& ABBOTT, L. (2006). The Enterobacteria. Journal American Society for Microbiology, 279-299. 
JANDA, J., ABBOTT, S., WOODWARD, D. \& KHASHE, S. (1998). Invasion of HEp-2 and other eukaryotic cell lines by Providencia: further evidence supporting the role of Providencia alcalifaciens in bacterial gastroenteritis. Curr Microbiol 37, 159-165.

MANOS, J. \& BELAS, R., (2006). The genera Proteus, Providencia, and Morganella. Procaryotes, 6, 245-269.

MURATA, T., LIDA, T., SHIOMI, Y., TAGOMORI, K., AKEDA, Y., YANAGIHARA, I., MUSHIAKE, S., ISHIGURO, F. \& Honda, T. (2001). A large outbreak of food borne infection attributed to Providencia alcalifaciens. Journal Infectious Disease 184, 1050-1055.

MARQUES, L., TAVECHIO, A., ABE, C. \& GOMES, T. (2003). Search for cytolethal distending toxin production among fecal Escherichia coli isolates fron brazilian children with diarrhea and without diarrhea. Journal of Clinical Microbiology. 45(5), 2206-2208.

PANDEY, M., KHAN, A., DAS, S., SARKAR, B., KAHALI, S., CHAKRABORTI, S., CHATTOPADHYAY, S., YAMASAKI, S., TAKEDA, Y., NAIR, B. \& RAMAMURHY, T. (2003). Association of cytothelal distending toxin locus $c d t B$ with enteropathogenic Escherichia coli isolated from patients with acute diarrea in Calcutta, India. Journal of Clinical Microbiology, 41, 5277-5281.

SENIOR, B. (1997). Media for the detection and recognition of the enteropathogen Providencia alcalifaciens in faeces. Journal of Medical Microbiology. 46, 524-527.
SHAH, M., ODOYO, E., LARSON, P., APONDI, E., KATHIIKO, C., MIRINGU, G., NAKASHIMA, M. \& ICHINOSE. Y. (2015). First report of a Foodborne Providencia alcalifaciens outbreak in Kenya. American Society of Tropical Medicine and Hygiene. doi:10.4269/ajtmh.15-0126.

SHIMA, A., HINENOYA, A., ASAKURA,M., NAGITA, A.\& YAMASAKI, S. (2012) Prevalence of Providencia Strains among Children with Diarrhea in Japan. Japanese Journal of Infectious Deseases, $65,545-547$.

SHIMA, A., HINENOYA, A., ASAKURA, M., SUGIMOTO, N., TSUKAMOTO, T., ITO, H., NAGITA, A., FARUQUE, S. \& YAMASAKIA, S., (2012). Molecular Characterizations of Cytolethal Distending Toxin Produced by Providencia alcalifaciens Strains Isolated from Patients with Diarrhea. Journal American Society for Microbiology, 80(4), 1323-1332.

SOBREIRA, M., LEAL, N., MAGHALAES, M., GUTH, B., ALMEIDA, A. (2001). Molecular analysis of clinical isolates of Providencia alcalifaciens. Journal Of Medical Microbiology, 50, 29-34.

YOH, M., MATSUMAYA, J., OHNISHI, M., TAGAKI, K., MIYAGI, H., MORI, K., PARK, K., ONO, T. \& HONDA, T. (2005). Importance of Providencia species as a major cause of travellers' diarrhoea. Journal of Medical Microbiology, 54, 1077-1082. 
